\title{
Scattering of Goldstone bosons and resonance production in a composite Higgs model on the lattice
}

\author{
Vincent Drach, ${ }^{a}$ Tadeusz Janowski, ${ }^{b}$ Claudio Pica ${ }^{c}$ and Sasa Prelovsek ${ }^{d, e, f}$ \\ ${ }^{a}$ Centre for Mathematical Sciences, Plymouth University, \\ Drake Circus, Plymouth, PL4 8AA, U.K. \\ ${ }^{b}$ The Higgs Centre for Theoretical Physics, The University of Edinburgh, \\ Peter Guthrie Tait Road, Edinburgh, EH9 3FD, U.K. \\ ${ }^{c}$ CP3-Origins and eScience Center, University of Southern Denmark, \\ Campusvej 55, DK-5230 Odense M, Denmark \\ ${ }^{d}$ Faculty of Mathematics and Physics, University of Ljubljana, \\ Jadranska 19, 1000 Ljubljana, Slovenia \\ e Jozef Stefan Institute, \\ Jamova 39, 1000 Ljubljana, Slovenia \\ ${ }^{f}$ Institute for Theoretical Physics, University of Regensburg, \\ 93040 Regensburg, Germany \\ E-mail: vincent.drach@plymouth.ac.uk, tadjanow@gmail.com, \\ pica@cp3-origins.net, sasa.prelovsek@ijs.si
}

ABStRaCT: We calculate the coupling between a vector resonance and two Goldstone bosons in $\mathrm{SU}(2)$ gauge theory with $N_{f}=2$ Dirac fermions in the fundamental representation. The considered theory can be used to construct a minimal Composite Higgs models. The coupling is related to the width of the vector resonance and we determine it by simulating the scattering of two Goldstone bosons where the resonance is produced. The resulting coupling is $g_{\mathrm{VPP}}=7.8 \pm 0.6$, not far from $g_{\rho \pi \pi} \simeq 6$ in QCD. This is the first lattice calculation of the resonance properties for a minimal UV completion. This coupling controls the production cross section of the lightest expected resonance at the LHC and enters into other tests of the Standard Model, from Vector Boson Fusion to electroweak precision tests. Our prediction is crucial to constrain the model using lattice input and for understanding the behavior of the vector meson production cross section as a function of the underlying gauge theory. We also extract the coupling $g_{\mathrm{VPP}}^{\mathrm{KSRF}}=9.4 \pm 0.6$ assuming the vector-dominance and find that this phenomenological estimate slightly overestimates the value of the coupling.

KEYwords: Lattice field theory simulation, Phenomenological Models

ArXiv EPrint: 2012.09761 


\section{Contents}

1 Introduction 1

2 Lattice setup $\quad 2$

3 Vector resonance from scattering of two pseudoscalars 5

3.1 Goldstone bosons scattering: flavour structure 6

$\begin{array}{lll}3.2 & \text { Operators and correlators } & 7\end{array}$

$\begin{array}{lll}3.3 & \text { Eigen-energies } & 7\end{array}$

$\begin{array}{lll}3.4 & \text { Scattering amplitude and phase shift } & 8\end{array}$

3.5 Mass $m_{R}$, width $\Gamma$ and the coupling $g_{\mathrm{VPP}}$ of the vector resonance 9

3.6 Comparison of the coupling $g_{\mathrm{VPP}}$ with the KSRF relation 11

4 Conclusions 11

$\begin{array}{ll}\text { A Conventions } & 12\end{array}$

\section{Introduction}

Among the numerous approaches to address the shortcomings of the Standard Model, new strongly coupled sectors provide a number of interesting mechanisms that address fundamental issues like the naturalness problem or the origin of the Higgs' field.

Pseudo Nambu-Goldstone Boson (PNGB) Composite Higgs models aim at identifying the Higgs degrees of freedom with the Goldstone bosons of a new strongly coupled sector [13]. In this framework, the fundamental Higgs field of the Standard Model is an effective low energy degree of freedom at the electroweak scale of a new strongly interacting sector featuring spontaneous chiral symmetry breaking. The mechanism alleviates the naturalness problem. The coupling of the new strongly interaction sector with the Standard Model breaks explicitly the flavour symmetry of the underlying gauge theory. This breaking is responsible for a non-trivial potential for the pseudo-Goldstone bosons which provides the Higgs with a mass and triggers electroweak symmetry breaking.

These models are constrained by comparing predictions at the electroweak scale with the LHC data. Typically the predictions depends on low energy constants (LECs) of the effective theory and on free parameters that are related to the model building itself. The vast majority of models are tested without specifying an underlying strongly interacting sector, and by assuming that the LECs are free parameters. While the approach allows to investigate entire classes of UV completion, it neglects correlations among LECs and additional information provided by a quantitative understanding of the strong dynamics. The lattice approach allows to make first-principle predictions of the low energy parameters and can therefore provide stringent constraints on some scenarios. 
Strongly interacting theories are expected to feature a rich spectrum of resonances that modify the phenomenology at colliders. In minimal scenarios, vector resonance are expected to mix with the electroweak bosons and can therefore be produced via vector boson fusion or via the Drell-Yan production mechanism, see for instance [4, 5]. These processes are controlled by the coupling $g_{\mathrm{VPP}}$ of the vector resonance $(V)$ to two pseudoscalar Goldstone bosons $(P)$. The phenomenology of new vector resonances is attracting considerable attention by the community [6-12].

In this work, we perform the first ab-initio calculation of the coupling $g_{\mathrm{VPP}}$ between a resonance and a pair of Goldstone bosons using lattice techniques in isolation of the Standard Model for a minimal UV completion. We consider SU(2) gauge theory with $N_{f}=2$ fundamental Dirac fermions. The theory features an extended SU(4) flavour symmetry that spontaneously breaks to $\mathrm{Sp}(4)$. It is used to build a PNGB Composite Higgs models in [13] and was recently reviewed in [14]. In this model the physical Higgs boson is a mixture of PNGBs and of the scalar state of the strong sector. The phenomenology of the model has been shown to be viable in view of the LHC data in [15], and the mixing between the scalar resonance and the Higgs can relax the bounds on the model [9]. The phenomenology of vector resonances for theories sharing the same chiral symmetry breaking pattern is investigated in detail as a function vector meson coupling constant in [16]. The authors derived the bound from di-lepton and di-boson searches at the LHC, as well as the dependence of the electroweak precision parameters as a function of the coupling constant. Our work can therefore be used to constrain a minimal model more efficiently.

We determine the $V \rightarrow P P$ coupling by extracting the scattering amplitude for $P P \rightarrow$ $V \rightarrow P P$ scattering in the vector channel via the rigorous Lüscher formalism $[17,18]$. We apply techniques that have been widely used for $\pi \pi \rightarrow \rho \rightarrow \pi \pi$ scattering in QCD, for example [19-25]. The coupling $g_{\mathrm{VPP}}$ is inferred from a Breit Wigner parametrization of the scattering amplitude. This work represents the first fully-fledged scattering calculation of the coupling $g_{\mathrm{VPP}}$ in a $4 \mathrm{D}$ gauge theory with fermions beyond QCD. It complements previous studies addressing the phenomenology of models based on the same underlying gauge theory [26-31].

The coupling $g_{\mathrm{VPP}}$ was previously estimated via the phenomenological relation $g_{\mathrm{VPP}}^{\mathrm{KSRF}}=m_{V} / F_{\mathrm{PS}}$ by Kawarabayashi-Suzuki-Riazuddin-Fayyazuddin (KSRF) $[32,33]$ that assumes the vector-meson dominance. These calculations were based on simulations where the vector state was stable. It was employed for QCD-like theories based on SU(3) [34, 35] as well as various groups $S U$ and $S p$ [35-39]. We refer to [34] for a discussion of the estimation of $g_{\mathrm{VPP}}^{\mathrm{KSRF}}$ for a number of gauge theories. This relation turns out to be satisfied in QCD but has however never been rigorously tested beyond before this work. It is therefore crucial to guide model builders and searches for Beyond the Standard Model Physics.

\section{Lattice setup}

In the numerical simulations of $\mathrm{SU}(2)$ gauge theory with $N_{f}=2$, we choose a cloverimproved Wilson action for the two flavours of Dirac fermions [40, 41] and the tree-level 
Symanzik improved action for the gauge sector [42]:

$$
\begin{aligned}
S= & -\frac{\beta}{2} \sum_{x, \mu, \nu} c_{0} \operatorname{Re} \operatorname{Tr} P_{\mu \nu}(x)+c_{1} \operatorname{Re} \operatorname{Tr}\left(R_{\mu \nu}(x)+R_{\nu \mu}(x)\right) \\
& +\sum_{x, \mu} \bar{\psi}(x)\left(a m_{0}+4\right) \psi(x)-\frac{1}{2} \bar{\psi}(x+\mu) U_{\mu}\left(1-\gamma^{\mu}\right) \psi(x)+\frac{c_{s w}}{2} \sum_{x, \mu<\nu} \bar{\psi}(x) \sigma_{\mu \nu} \hat{F}^{\mu \nu} \psi(x) .
\end{aligned}
$$

Here $U_{\mu}$ is the gauge field, $\psi$ is the doublet of $u$ and $d$ fermions, $P_{\mu \nu}$ is the plaquette, $R_{\mu \nu}$ is a $2 \times 1$ rectangular loop, $\hat{F}_{\mu \nu}$ is the Wilson clover term and $\beta$ is the inverse lattice gauge coupling. The coefficient of the gauge action are set to $c_{0}=5 / 3$ and $c_{0}+8 c_{1}=1$ [42]. The improvement coefficient $c_{S W}=1$ is set to its tree-level value in our simulations. The presence of bare mass term $a m_{0}$ and the Wilson term explicitly breaks the SU(4) flavour symmetry to a $\mathrm{Sp}(4)$ subgroup. We use periodic boundary conditions in all four directions for the fermions.

The results presented in this work are obtained at $\beta=1.45$. For each ensemble, we compute the pseudoscalar meson mass $m_{\mathrm{PS}}$, and vector meson mass $m_{\mathrm{V}}$ by fitting at large euclidean time the effective mass of appropriate two-points correlation function. We also determine the bare quark mass $m_{\mathrm{PCAC}}$ defined through the Partially Conserved Axial Current relation and the bare pseudoscalar decay constant $F_{\mathrm{PS}}^{\mathrm{bare}}$, which renormalizes multiplicatively with the renormalization factor $Z_{A}$. The normalization of $F_{\mathrm{PS}}$ corresponds to the convention where the pion decay constant in QCD is $130 \mathrm{MeV}$. More details concerning our calculation of these quantities can be found in [26] where we use the same strategy and normalization.

To compare our results with our previous results we convert the lattice quantities in physical units by setting the scale using the Wilson Flow observable $w_{0}$ [43]. The scale $w_{0}$ is defined by $W\left(w_{0}^{2}\right)=W_{\text {ref }}$, where $W(t)=t \frac{d}{d t}\left[t^{2} E(t)\right]$ and $E(t)^{1}$ is the flowed action density at flow time $t$. The reference value $W_{\text {ref }}$ is dimensionless and set to 1.0 , consistently with our previous definition. The value of $w_{0} / a$ is determined for a range of fermion masses and chirally extrapolated using NNLO expansion in terms of $m_{\mathrm{PS}}^{2}$ [44]. We employ ensembles with $m_{\mathrm{PS}} L>4$, where the finite volume effects are small. The data and are shown in figure 1 , together with the best fitting curve, and the $1 \sigma$ error band in gray. The final results reads $w_{0}^{\chi} / a(\beta=1.45)=3.08(2)$ using the NNLO fit which has a $\chi^{2} / n d o f=6.5 / 4$.

In order to compare our result with the KSRF relation $g_{\mathrm{VPP}}=m_{V} / F_{\mathrm{PS}}$, it is necessary to renormalize the pseudoscalar decay constant with $Z_{A}$. We performed the non perturbative determination of $Z_{A}$ in the RI'-MOM scheme [45], using the same strategy as in our previous setup [26]. The correlators are estimated using momentum sources introduced in [46]. To interpolate between the lattice momenta we furthermore use twisted boundary conditions $[47,48]$. The amputated vertex function is then defined as follows:

$$
\Pi_{\mu}(p)=S(p)^{-1}\left(\sum_{x, y, z} e^{-i p(x-z)} e^{-i p(z-y)}\left\langle\psi(x) \bar{\psi}(z) \gamma_{\mu} \gamma_{5} \psi(z) \bar{\psi}(y)\right\rangle\right) S(p)^{-1}
$$

\footnotetext{
${ }^{1} E$ and $t$ are not to be confused with energy and Eucledian time employed later on.
} 


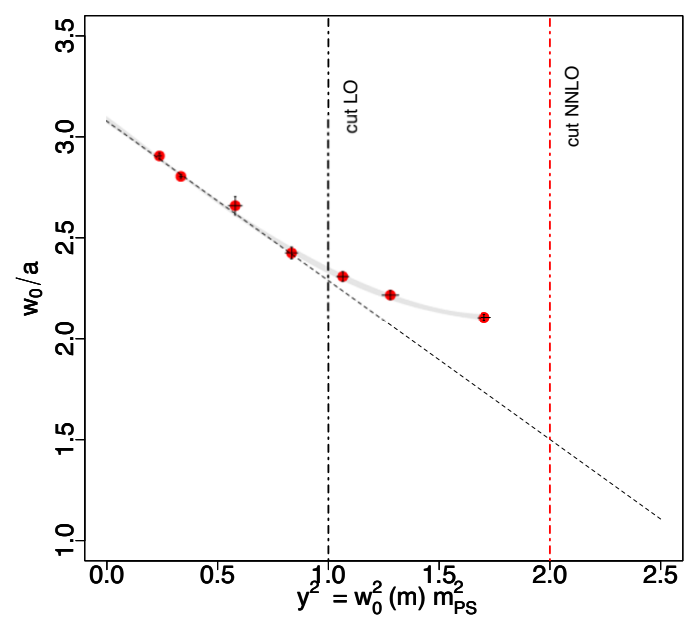

Figure 1. Chiral extrapolation of $w_{0} / a$ as a function of $y^{2}=w_{0}(m)^{2} m_{\mathrm{PS}}^{2}$. The lattice data are in red. The LO fit is represented by a dotted straight line and the NNLO fit is represented by a shaded gray area. The vertical dotted line denotes the maximal value of $y^{2}$ included in the LO and NNLO fits.

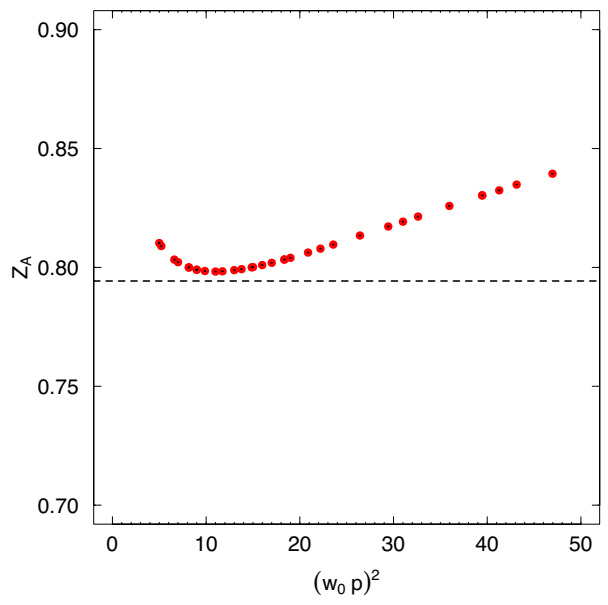

Figure 2. Behaviour of $Z_{A}$ as a function of $\left(w_{0} p\right)^{2}$ after the chiral extrapolation of $\Lambda_{A}$. The statistical errors are included. The perturbative value derived for the plaquette action and unimproved Wilson fermions [49] is shown by a black dashed line.

where $S^{-1}(p)$ is the inverse propagator in spin and color space. Defining,

$$
\Lambda_{A}\left(p^{2}\right)=-i \frac{\operatorname{tr}\left[\frac{\gamma_{\mu} \sin \left(a p_{\mu}\right)}{\sin ^{2}\left(a p_{\mu}\right)} S^{-1}(p)\right]}{\sum_{\mu} \operatorname{tr}\left[P_{\mu} \Pi_{\mu}(p)\right]}, \quad \text { with } \quad P_{\mu}=\frac{\gamma_{\mu} \gamma_{5}}{4},
$$

it can be shown that in the chiral limit $\Lambda_{A}\left(p^{2}=\mu^{2}\right) \longrightarrow Z_{A}\left(\beta, \mu^{2}\right)$. The chiral extrapolation at fixed $p^{2}$ is performed using linear extrapolation in $m_{\mathrm{PCAC}}$ and our estimate of $\Lambda_{A}\left(p^{2}\right)$ in the chiral limit is shown in figure 2. We used $\left(w_{0}^{\chi} p\right)^{2}=7>\left(w_{0}^{\chi} m_{V}\right)^{2} \sim 1$ as a reference scale and our final estimate reads $Z_{A}\left(\beta=1.45,\left(w_{0}^{\chi} p\right)^{2}=7\right)=0.8022(3)$. The statistical errors are estimated using bootstrap resampling. 


\begin{tabular}{|ccc|cc|cccc|}
\hline$\beta$ & $m_{0}$ & $V$ & Stat. & $m_{\mathrm{PS}} L$ & $a m_{\mathrm{PCAC}}$ & $a m_{\mathrm{PS}}$ & $a F_{\mathrm{PS}}^{\text {bare }}$ & $a m_{V}^{\text {naive }}$ \\
\hline 1.45 & -0.605 & $16^{3} \times 32$ & 1354 & 3.6 & $0.0107(7)$ & $0.226(4)$ & $0.048(2)$ & $0.488(42)$ \\
1.45 & -0.605 & $24^{3} \times 48$ & 1857 & 4.9 & $0.0107(2)$ & $0.205(2)$ & $0.058(1)$ & $0.438(26)$ \\
\hline
\end{tabular}

Table 1. The main parameters of two lattice ensembles employed for the study of the vector resonance. The values obtained for $a m_{\mathrm{PCAC}}, a m_{\mathrm{PS}}, a F_{\mathrm{PS}}$ and $a m_{V}^{\text {naive }}$ are also provided.

A range of simulations performed at $\beta=1.45$ allowed us to determine simulation parameters so that $m_{\mathrm{V}}>2 m_{\mathrm{PS}}$, therefore allowing the decay of the vector meson into two Goldstone bosons. We used a single bare mass $m_{0}=-0.6050$ to perform the calculation of the phase-shift of the two Goldstone boson scattering process, and two lattice volumes of $16^{3} \times 32$ and $24^{3} \times 48$. The value of the $m_{\mathrm{PS}}, m_{\mathrm{PCAC}}$ and $m_{\mathrm{V}}^{\text {naive }}$ are summarized in table 1. The topological charge was monitored during the runs, our simulations explore a range of topological sectors. In average the topological charge is compatible with zero. The scattering study employs the quark propagators that are combined from the propagators with periodic and anti-periodic boundary conditions in time in order to effectively double the time extent of the lattice, as detailed for example in [22].

\section{Vector resonance from scattering of two pseudoscalars}

The vector resonance $V$ can decay into two pseudoscalar mesons $P$ as shown based on the symmetries in the next subsection. The vector meson mass extracted from the twopoint correlator on the chosen gauge ensemble is above the decay threshold $2 m_{\mathrm{PS}}$ and the decay is therefore kinematically allowed. A resonance is not an eigenstate of the Hamiltonian and its properties have to be inferred from the study of the scattering process $P P \rightarrow V \rightarrow P P$. We will show that the investigation of this resonance has similarities with the study of the $\rho$-vector resonance in QCD which has been studied through $\pi \pi \rightarrow \rho \rightarrow \pi \pi$ in numerous lattice QCD investigations, for example [19-25]. The main strategy is briefly reviewed here. The discrete eigen-energies $E_{n}$ of two pseudoscalars in the finite box of size $L$ and with periodic boundary conditions in space are determined. These are different from the non-interacting energies $E^{n . i .}=\left(m_{\mathrm{PS}}^{2}+\mathbf{p}_{1}^{2}\right)^{1 / 2}+\left(m_{\mathrm{PS}}^{2}+\mathbf{p}_{2}^{2}\right)^{1 / 2}$ of two pseudoscalars with momenta $\mathbf{p}_{1,2}=\frac{2 \pi}{L} \mathbf{n}_{1,2}$ due to mutual interactions of pseudoscalars. The energyshifts $\Delta E=E_{n}-E^{n . i}$ give information on their interactions and therefore phase-shift in partial wave $l$. Lüscher's formalism provides a rigorous relation between the eigenenergy $E_{C M}$ of two-particles in a finite box and their infinite-volume scattering amplitude $S_{l}\left(E_{C M}\right)=e^{2 i \delta_{l}\left(E_{C M}\right)}$ at this energy $E_{C M}$ in center-of-momentum frame [17, 50]. We will extract the phase shift for seven values of energy by considering the lowest two eigenstates $E_{n=1,2}$ in two different inertial frames and two different volumes. ${ }^{2}$ The energy-dependence of the phase shift will have a resonance shape. The resonance mass, width and the $V \rightarrow P P$ coupling $g_{\mathrm{VPP}}$ will be extracted from this dependence via the Breit-Wigner type fit.

\footnotetext{
${ }^{2}$ This renders eight values of $E_{C M}$ and $\delta\left(E_{C M}\right)$, but one of them will have large uncertainty and will not be employed.
} 


\subsection{Goldstone bosons scattering: flavour structure}

We consider the scattering channels of the two-Goldstone boson scattering in $\mathrm{SU}(2)$ gauge theory with $N_{f}=2$ fundamental fermions. The goal of this section is to show that one can employ the interpolators with the flavor structure analogous to QCD. For this purpose we demonstrate that the operators $\pi^{-} \pi^{+}-\pi^{+} \pi^{-}$and $\rho_{\mu}$ belong to the ten-dimensional irreducible representation of $\operatorname{Sp}(4)$, where $\pi$ and $\rho$ are bilinears

$$
\pi^{+}=-\bar{d} \gamma_{5} u, \quad \pi^{-}=\bar{u} \gamma_{5} d, \quad \rho_{\mu}=\bar{u} \gamma_{\mu} u-\bar{d} \gamma_{\mu} d
$$

We follow the convention used for instance in [51] and summarized in appendix A, where

$$
Q=\left(\begin{array}{c}
u_{L} \\
d_{L} \\
\widetilde{u}_{L} \\
\widetilde{d}_{L}
\end{array}\right)=\left(\begin{array}{c}
u_{L} \\
d_{L} \\
\left(-i \sigma_{2}\right) C \bar{u}_{R}^{T} \\
\left(-i \sigma_{2}\right) C \bar{u}_{R}^{T}
\end{array}\right), \quad E=\left(\begin{array}{cc}
0 & \mathbb{1}_{2} \\
-\mathbb{1}_{2} & 0
\end{array}\right) \text {, }
$$

the Pauli matrix acts in colour space and $C=i \gamma_{0} \gamma_{2}$ is the conjugation charge matrix. It can be shown that the massless Lagrangian is symmetric under $\mathrm{SU}(4)$ transformation, while the mass term can be shown to be $\mathrm{Sp}(4)$ invariant.

Let's focus on the multiplet $\Pi^{i=1, \ldots, 5}$ and the decoupled $V_{\mu}^{a=1, \ldots, 10}$ defined as

$$
\Pi^{i}=Q^{T}\left(-i \sigma_{2}\right) C \gamma_{5} X^{i} E Q+\left(Q^{T}\left(-i \sigma_{2}\right) C \gamma_{5} X^{i} E Q\right)^{\dagger}, \quad V_{\mu}^{a}=\bar{Q} S^{a} \gamma_{\mu} Q
$$

Here $X^{i=1, \ldots, 5}$ are the broken generators used to parametrized the coset $\mathrm{SU}(4) / \mathrm{Sp}(4)$, while $S^{a=1, \ldots, 10}$ are the generators of $\operatorname{Sp}(4)$ defined in the appendix.

Consider an infinitesimal the infinitesimal transformation $Q \longrightarrow\left(\mathbb{1}_{4}+i \alpha^{a} S^{a}\right) Q$ where $\alpha^{i=1, \ldots, 10}$ are real parameters. The explicit calculation shows that the multiplet $\boldsymbol{\Pi}$ transforms as $\boldsymbol{\Pi} \longrightarrow \boldsymbol{\Pi}+G \boldsymbol{\Pi}$, where $G=2 i \sqrt{2} \alpha^{a} T^{a}$ is a skew-symmetric matrix which can be written as a linear combination of the generators $T^{a=1, \ldots, 10}$ of $\mathrm{SO}(5)$ defined in the appendix. The multiplet $\Pi$ therefore transforms as a 5 -dimensional irreducible representation of $\operatorname{Sp}(4)$.

The transformation of the tensor product of two Goldstone Bosons operators $\Pi \otimes \boldsymbol{\Pi}$ can then be worked out. In this work, we focus on the antisymmetric part of the tensor:

$$
H^{i j}=\frac{1}{2}\left(\Pi^{i} \Pi^{j}-\Pi^{i} \Pi^{j}\right) .
$$

Defining the 10-dimensional vector $F^{a}=\operatorname{tr}\left[T^{a} H\right]$, an explicit calculation shows that $F^{a} \longrightarrow F^{a}+2 i f_{a b c} \alpha^{b} F^{c}$, where $f_{a b c}$ are the structure constant of $\operatorname{Sp}(4)$. The multiplet $F^{a=1, \ldots, 10}$ therefore belongs to a 10 -dimensional irreducible representation of $\mathrm{Sp}(4)$. Furthermore, expressing eq. (3.3) in terms of the $u$ and $d$ fields via eq. (3.2), using properties of the conjugation charge matrix and of the skew-symmetric matrix $\left(-i \sigma_{2}\right)$ acting in color space, we find that $F^{3}=\frac{1}{4}\left(\pi^{-} \pi^{+}-\pi^{+} \pi^{-}\right)$. In a similar manner the bilinears $V_{\mu}^{a}$ transform as $V^{a} \longrightarrow V^{a}+i f_{a b c} \alpha^{b} S^{c}$ and $V_{\mu}^{3}=\rho_{\mu}$. 
In other words, we have shown that the usual 3-dimensional irrep $(I=1)$ of the isospin group is contained in the 10-dimensional representation of $\mathrm{Sp}(4)$. Regarding discrete symmetries, it straightforward to check that $F^{3}$ and $\rho_{\mu}$ have negative parity and negative charge conjugation.

\subsection{Operators and correlators}

The aim is to extract eigen-energies of the system with the quantum numbers of the vector resonance, namely $J^{P}=1^{-}$. Given the flavour structure discussed in the previous section, we employ the two following operators:

$$
\begin{aligned}
O_{P P}(t, \mathbf{p}, \mathbf{0})=O_{1} & =\frac{1}{\sqrt{2}} \sum_{\mathbf{x}} \bar{d}(x) \gamma^{5} u(x) e^{i \mathbf{p} \cdot \mathbf{x}} \sum_{y} \bar{u}(y) \gamma^{5} d(y) e^{i \mathbf{y} \cdot \mathbf{0}}-\{u \leftrightarrow d\} \\
& \propto P^{+}(\mathbf{p}) P^{-}(0)-P^{-}(\mathbf{p}) P^{+}(0) \\
O_{V}(t, \mathbf{p})=O_{2} & =\frac{1}{\sqrt{2}} \sum_{\mathbf{x}} \bar{u}(x)\left(\gamma \cdot \frac{\mathbf{p}}{|\mathbf{p}|}\right) u(x) e^{i \mathbf{p} \cdot \mathbf{x}}-\{u \leftrightarrow d\} .
\end{aligned}
$$

To compute the phase shift for different values of $E_{C M}$ we consider two inertial frames with total momenta $\mathbf{P}_{\text {tot }}=\mathbf{p}=(0,0,1)$ and $(1,1,0)$ in units of $2 \pi / L$. The operators with momenta $(0,0,1)$ transform according to the irreducible representation $A_{2}^{-}$of the group $D_{4 h}$ within the notation of [18]. The operators with momenta $(1,1,0)$ transform according to $B_{1}^{-}$of the group $D_{2 h}$ in the notation of [21].

The eigen-energies are obtained by computing in each frame the $2 \times 2$ correlation matrix

$$
C(t, \mathbf{p})=\left(\begin{array}{cc}
\left\langle O_{P P}(t, \mathbf{p}, \mathbf{0}) O_{P P}^{\dagger}(0, \mathbf{p}, \mathbf{0})\right\rangle & \left\langle O_{P P}(t, \mathbf{p}, \mathbf{0}) O_{V}^{\dagger}(0, \mathbf{p})\right\rangle \\
\left\langle O_{V}(t, \mathbf{p}) O_{P P}^{\dagger}(0, \mathbf{p}, \mathbf{0})\right\rangle & \left\langle O_{V}(t, \mathbf{p}) O_{V}^{\dagger}(0, \mathbf{p}, \mathbf{0})\right\rangle
\end{array}\right),
$$

which renders the Wick contractions analogous to those in QCD. We evaluate those with the stochastic $\mathrm{U}(1)$ noise technique used in simulations [19, 20] of $\rho$-meson decay in QCD.

The experience from analogous studies of $\pi \pi \rightarrow \rho \rightarrow \pi \pi$ scattering in QCD indicates that it is crucial to employ one $\bar{q} q$ operator and the $\pi \pi$ operators relevant in the given energy range to obtain eigen-energies of the system. Adding more $\bar{q} q$ operators with given quantum numbers does not affect the eigen-energies, as illustrated for example in figure 8 of [22]. Therefore we expect that two employed operators suffice to extract eigen-energies in this energy range.

\subsection{Eigen-energies}

The time-dependence of the correlation matrix $C_{i j}(t)=\left\langle 0\left|O_{i}(t) O_{j}^{\dagger}(0)\right| 0\right\rangle=$ $\sum_{n}\left\langle 0\left|O_{i}\right| n\right\rangle e^{-E_{n} t}\left\langle n\left|O_{j}^{\dagger}\right| 0\right\rangle$ contains information on the eigen-energies $E_{n}$. We extract those using the widely used GEVP variational method [52-54]

$$
C(t) u^{(n)}(t)=\lambda_{n}(t) C\left(t_{0}\right) u^{(n)}\left(t_{0}\right), \quad \lambda_{n}(t) \stackrel{\text { large } t}{\longrightarrow} A_{n} e^{-E_{n} t}
$$

with reference time $t_{0}=4$. 

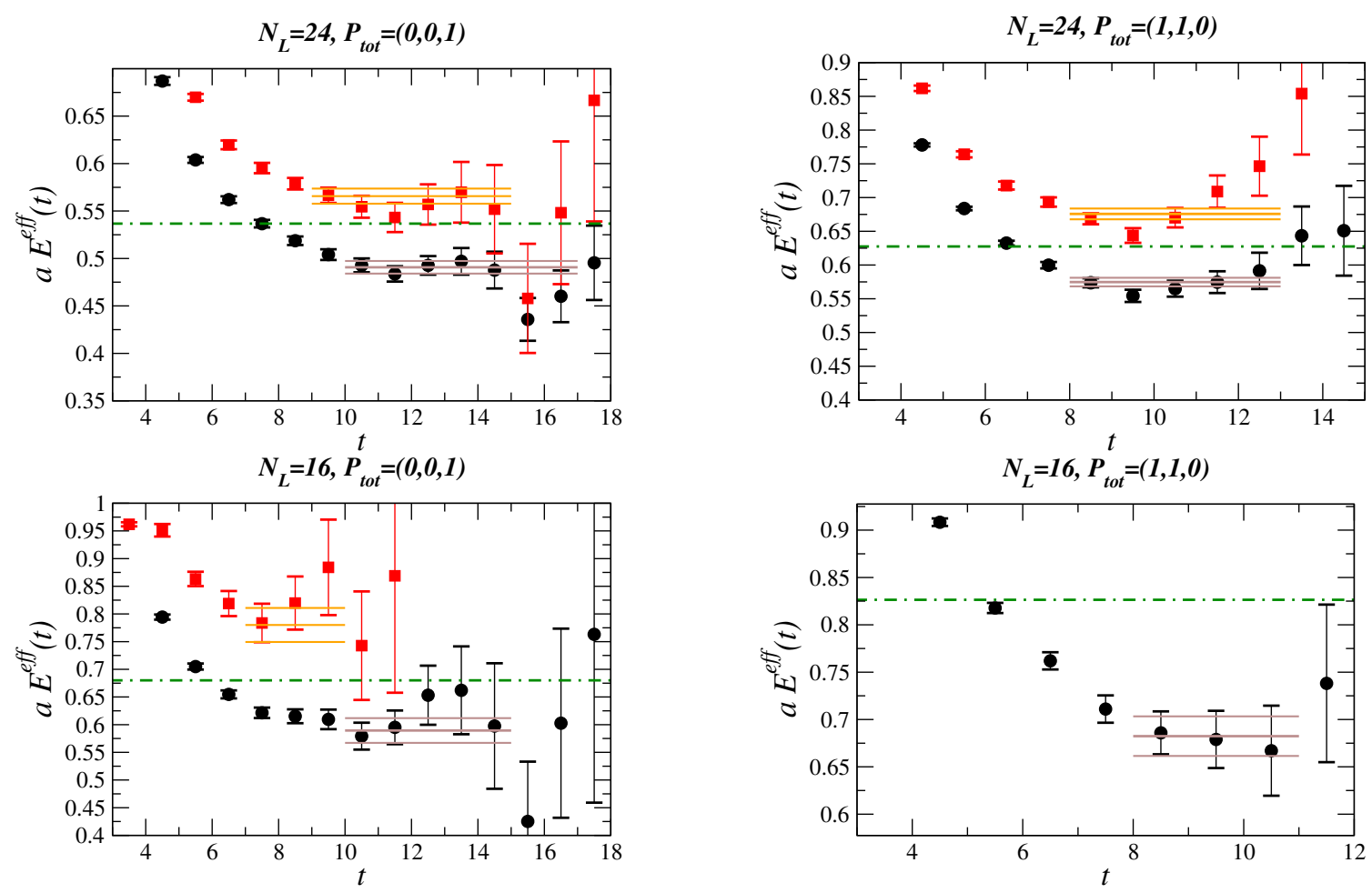

Figure 3. The effective energies $E_{n}^{\text {eff }}(t)$ for seven eigenstates of the two-pseudoscalar system on a finite lattice. The fitted eigen-energies $E_{n}$ are also provided together with the fit-ranges (3.7). The dot-dashed lines indicate non-interacting energy $E^{n . i .}=\left(m_{\mathrm{PS}}^{2}+\mathbf{p}^{2}\right)^{1 / 2}+m_{\mathrm{PS}}$ of two -pseudoscalar system $P(0) P(\mathbf{p})$.

The effective energies $E_{n}^{\mathrm{eff}}(t)=\log \left[\lambda_{n}(t) / \lambda_{n}(t+1)\right]$ for seven eigenstates are given in figure 3 and they are related to $E_{n}$ in the plateau region. They correspond to lowest two eigenstates for two inertial frames and two volumes; the first excited state for $N_{L}=16$ and $\mathbf{P}_{\text {tot }}=(1,1,0)$ has a large statistical uncertainty and will not be used in analysis. We do not find an indication for non-exponential time-dependence at large $t$ before the signal is lost in the noise since the size of the time-direction has been effectively doubled by combining periodic and antiperiodic propagators in time. The eigen-energies $E_{n}$ are extracted from the correlated one-exponential fits (3.7) of eigenvalues $\lambda_{n}(t)$ in the plateau region, that are indicated in the figure 3 and tabulated in 2.

\subsection{Scattering amplitude and phase shift}

The eigen-energies $E_{n}$ in figure 3 (solid lines) are shifted with respect to non-interacting energy $E^{n . i .}=\left(m_{\mathrm{PS}}^{2}+\mathbf{p}^{2}\right)^{1 / 2}+m_{\mathrm{PS}}$ of two-pseudoscalar system $P(\mathbf{p}) P(0)$ (dot-dashed lines). These non-zero shifts are essential for extracting the scattering information.

The relation between eigen-energy $E_{C M}$ of two particles and infinite-volume scattering phase shift $\delta\left(E_{C M}\right)$ at that energy was derived by Lüscher [17] for $\mathbf{P}_{\text {tot }}=0$. This relation was generalized in $[18,21]$ for inertial frames with $\mathbf{P}_{\text {tot }}=(0,0,1)$ and $(1,1,0)$ that are employed here. In this case, the eigenstate with energy $E_{n}$ corresponds to the 


\begin{tabular}{|ccc|cccccc|}
\hline$N_{L}$ & $\mathbf{P}_{\text {tot }}$ & level $n$ & fit-range & $E_{n} a$ & $E_{C M} a$ & $\left(\frac{p_{*} L}{2 \pi}\right)^{2}$ & $\frac{\left(p_{*} a\right)^{3} \cot \delta_{1}}{E_{C M} a}$ & $\left.\delta_{l=1}{ }^{\circ}\right]$ \\
\hline 24 & $(0,0,1)$ & 1 & $10-17$ & $0.491(7)$ & $0.415(8)$ & $0.016(23)$ & $0.0055(11)$ & $0.9(1.4)$ \\
24 & $(0,0,1)$ & 2 & $9-15$ & $0.566(8)$ & $0.501(9)$ & $0.30(3)$ & $-0.027(5)$ & $167(4)$ \\
24 & $(1,1,0)$ & 1 & $8-13$ & $0.575(6)$ & $0.440(8)$ & $0.093(27)$ & $0.0045(11)$ & $14.3(2.2)$ \\
24 & $(1,1,0)$ & 2 & $8-13$ & $0.676(8)$ & $0.565(9)$ & $0.55(4)$ & $-0.025(3)$ & $152(4.9)$ \\
16 & $(0,0,1)$ & 1 & $10-15$ & $0.589(22)$ & $0.440(30)$ & $-0.020(42)$ & $0.013(3)$ & $-1.7(5.8) \mathrm{i}$ \\
16 & $(0,0,1)$ & 2 & $7-10$ & $0.780(31)$ & $0.674(36)$ & $0.40(8)$ & $-0.044(8)$ & $152(10)$ \\
16 & $(1,1,0)$ & 1 & $8-11$ & $0.682(21)$ & $0.397(36)$ & $-0.078(46)$ & $0.0085(17)$ & $-24(20) \mathrm{i}$ \\
\hline
\end{tabular}

Table 2. Seven eigen-states of $P P$ system and the resulting information related to the scattering phase shift $\delta_{l}$ for partial-wave $l=1$.

energy $E_{C M}=\left(E_{n}^{2}-\mathbf{P}_{\text {tot }}^{2}\right)^{1 / 2}$ in the center-of-momentum frame, where pseudoscalars $P\left(\mathbf{p}_{*}\right) P\left(-\mathbf{p}_{*}\right)$ have back-to-back momenta $p_{*}=\left(E_{C M}^{2} / 4-m_{\mathrm{PS}}^{2}\right)^{1 / 2}$ and dimension-less momenta $q=p_{*} L /(2 \pi)$. The relation between eigen-energy (or corresponding $q$ ) and the scattering phase-shift at that energy (or $q$ ) is given by

$$
\begin{aligned}
& \tan \delta_{1}(q)=\frac{\pi^{3 / 2} q \gamma}{Z_{00}\left(1 ; q^{2}\right)+\frac{2}{\sqrt{5} q^{2}} Z_{20}\left(1 ; q^{2}\right)} \quad \text { for } \mathbf{P}_{\text {tot }}=(0,0,1) \\
& \tan \delta_{1}(q)=\frac{\pi^{3 / 2} q \gamma}{Z_{00}\left(1 ; q^{2}\right)-\frac{1}{\sqrt{5} q^{2}} Z_{20}\left(1 ; q^{2}\right)-i \frac{\sqrt{3}}{\sqrt{10} q^{2}}\left(Z_{22}\left(1 ; q^{2}\right)-Z_{2(-2)}\left(1 ; q^{2}\right)\right)} \quad \text { for } \mathbf{P}_{\text {tot }}=(1,1,0),
\end{aligned}
$$

for the relevant irreducible representations of the symmetry groups listed after eq. (3.5). Here $\delta_{1}$ denotes phase shift for partial-wave $l=1, Z_{l m}\left(s, q^{2}\right)=\sum_{n \in P_{d}} Y_{l m}(n) /\left(q^{2}-n^{2}\right)^{s}[21]$ and $\gamma=\left(1-v^{2}\right)^{-1 / 2}$.

Every eigen-energy $E_{n}$ renders certain $E_{C M}, q$ and phase shift, which are tabulated for seven eigenstates in table 2. The dependence of the phase-shift on $E_{C M}$ is plotted in figure $4 \mathrm{a}$. It increases from the small values, past the resonance value $90^{\circ}$ to the large values.

\subsection{Mass $m_{R}$, width $\Gamma$ and the coupling $g_{\mathrm{VPP}}$ of the vector resonance}

The energy dependence of the phase shift for $P P$ scattering in figure 4a shows a behavior expected for a narrow resonance that lies slightly above threshold: it rises from small values, through the resonance value $90^{\circ}$ at about $m_{R} a=E_{C M} a \simeq 0.45$, to the large values close to $180^{\circ}$. It is expected to have a Breit-Wigner type resonance form in the vicinity of a narrow vector resonance

$$
\tan \delta_{1}\left(E_{C M}\right)=\frac{E_{C M} \Gamma\left(E_{C M}\right)}{m_{R}^{2}-E_{C M}^{2}}, \quad \Gamma\left(E_{C M}\right)=\frac{g_{\mathrm{VPP}}^{2}}{6 \pi} \frac{p_{*}^{3}}{E_{C M}^{2}} .
$$

The resonance width $\Gamma\left(E_{C M}\right)$ is parametrized in terms of the $V \rightarrow P P$ coupling $g_{\mathrm{VPP}}$ and the phase space for partial wave $l=1$, where $p_{*}$ is the momentum of the pseudoscalar in $\mathrm{CM}$ frame. The resonance phase shift (3.9) implies that $p_{*}^{3} \cot \delta_{1} / E_{C M}$ is a linear function 

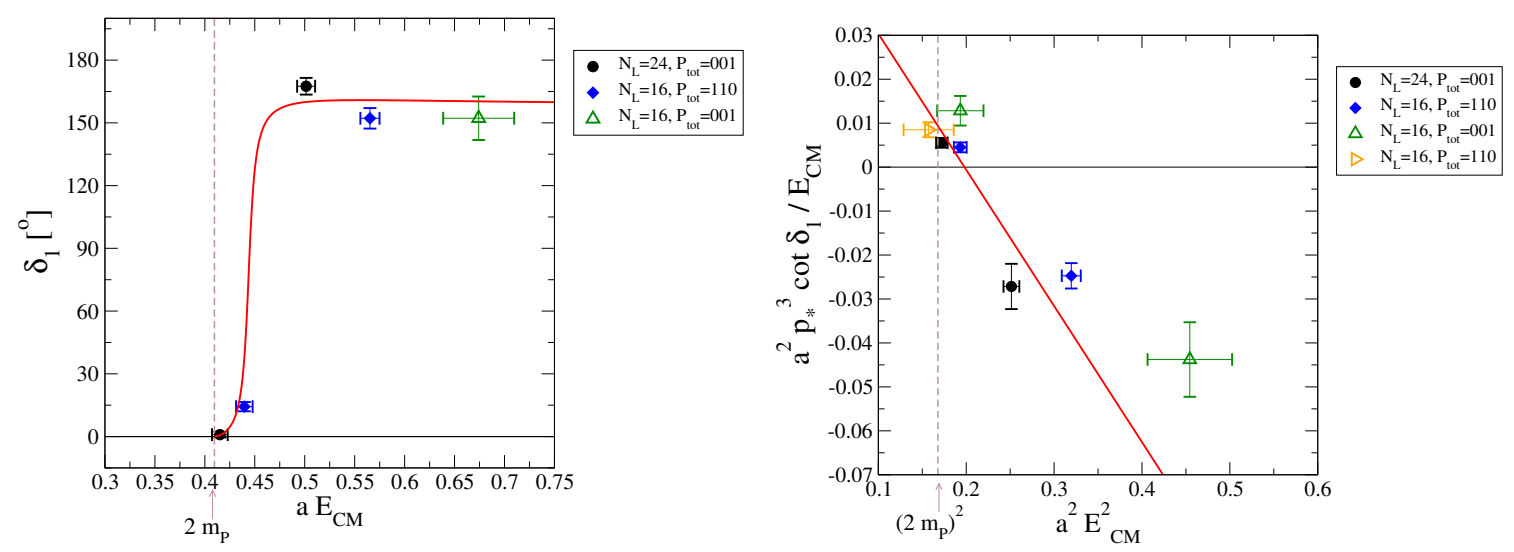

Figure 4. Results on the scattering $P P$ of two pseudoscalars in partial-wave $l=1$. (a) Scattering phase-shift $\delta_{1}$ as a function of center-of-momentum energy $E_{C M}$. (b) Quantity $p_{*}^{3} \cot \delta_{1}\left(E_{C M}\right) / E_{C M}$ as a function of $E_{C M}^{2}$, which is expected to be linear in case of Breit-Wigner resonance. The red solid line shows the Breit-Wigner resonance fit (3.9), (3.10) with the values of resonance parameters (3.12). Dashed vertical line indicates the position of the threshold for $N_{L}=24$. The left plot omits the points below threshold that lead to imaginary phase shifts.

of $E_{C M}^{2}$

$$
\frac{p_{*}^{3} \cot \delta_{1}\left(E_{C M}\right)}{E_{C M}}=\frac{6 \pi}{g_{\mathrm{VPP}}^{2}}\left(m_{R}^{2}-E_{C M}^{2}\right)
$$

This quantity is shown in figure $4 \mathrm{~b}$ for $P P$ scattering from our lattice simulation. It is falling with energy, it crosses through the resonance value $\cot \delta_{1}=0$ at $E_{C M}=m_{R}$ and roughly supports the linear dependence on $E_{C M}^{2}$.

Finally, we proceed to determine the vector resonance parameters from the phase shift that shows a resonant shape. We aim to determine the mass $m_{R}$ and the coupling $g_{\mathrm{VPP}}$ that parametrizes the width rather than the width itself. The width is strongly dependent on $m_{\mathrm{PS}}$ through the phase space, while the dependence of $g_{\mathrm{VPP}}$ on $m_{\mathrm{PS}}$ is expected to be much milder (the dependence of $g_{\rho \pi \pi}$ on $m_{\pi}$ in QCD is very mild as evidenced in the review of various lattice results $[24,25])$. The $m_{R}$ and $g_{\mathrm{VPP}}$ can be read-off from zero and the slope for the quantity (3.10) in figure $4 \mathrm{~b}$. In order to take the correlations properly into account, we extract $m_{R}$ and $g_{\mathrm{VPP}}$ by minimizing the correlated $\chi^{2}$

$$
\chi^{2}=\sum_{k=1}^{N_{k}} \sum_{k^{\prime}=1}^{N_{k}}\left[E_{k}-E_{k}^{p a r}\left(m_{R}, g_{\mathrm{VPP}}\right)\right] \operatorname{Cov}^{-1}\left(k, k^{\prime}\right)\left[E_{k^{\prime}}-E_{k^{\prime}}^{p a r}\left(m_{R}, g_{\mathrm{VPP}}\right)\right]
$$

with $N_{k}=7$ for seven eigenstates. Here $E_{k=1, \ldots, N_{k}}$ are energies of seven eigenstates determined on the lattice. $\operatorname{Cov}\left(k, k^{\prime}\right)$ is their $7 \times 7$ covariance matrix, where $\operatorname{Cov}\left(k, k^{\prime}\right)=0$ if eigenstates $k$ and $k^{\prime}$ correspond to ensembles with different $N_{L}$. The $E_{k}^{p a r}\left(m_{R}, g_{\mathrm{VPP}}\right)$ is the value of $E_{k}$ determined analytically for given $m_{R}$ and $g_{\mathrm{VPP}}$ based on the parametrization (3.10) and $\delta_{1}$ from Lüscher-type relation (3.8). 
The final parameters of the vector resonance based on minimization of $\chi^{2}(3.11)$ are

$$
\begin{aligned}
& m_{R} a=0.445 \pm 0.008 \pm 0.002 \\
& g_{\mathrm{VPP}}=7.8 \pm 0.5 \pm 0.1 \\
& \text { with } \quad \operatorname{cor}\left(m_{R}, g_{\mathrm{VPP}}\right)=0.35 \\
& \text { and } \quad \chi^{2} / \text { dof }=1.5 \text {. }
\end{aligned}
$$

The first error is statistical and arises from the errors on the energies encoded in the covariance matrix, while the second error accounts for the uncertainty in the pseudoscalar mass. The correlation between resonance parameters is provided by cor $=$ $\frac{\left\langle(g-\langle g\rangle)\left(m_{R}-\left\langle m_{R}\right\rangle\right)\right\rangle}{\sqrt{\left\langle(g-\langle g\rangle)^{2}\right\rangle} \sqrt{\left\langle\left(m_{R}-\left\langle m_{R}\right\rangle\right)^{2}\right\rangle}}$. The phase-shift dependence based on these resonance parameters (3.12) is plotted by red solid lines in figure 4. The results (3.12) are based on seven eigenstates from both volumes $N_{L}=24,16$. Restricting the fit to four eigenstates on the larger volume $N_{L}=24$, one gets compatible results $m_{R} a=0.442 \pm 0.009 \pm 0.002, g_{\mathrm{VPP}}=$ $7.7 \pm 0.5 \pm 0.1$ with cor $=0.37$ and $\chi^{2} / d o f=1.7$. For comparison, table 1 presents the mass of a vector particle ( $\left.a m_{V}^{\text {naive }}\right)$ obtained using a single $\bar{q} q$ operator in a conventional way.

Note that the calculation is performed at the finite pseudoscalar meson mass and at finite lattice spacing. However it should be noted that lattice results on $g_{\rho \pi \pi}$ in QCD do not show large discretization errors and that its dependence on $m_{\pi}$ is very mild: the compilation $[24,25]$ of lattice simulations by several groups shows that the coupling is $g_{\rho \pi \pi} \simeq 6$ for a wide range of $m_{\pi}$ and lattice spacings. We therefore believe our calculation provides a first estimate of the coupling $g_{\mathrm{VPP}}$ in the chiral limit of SU(2) gauge theory with two fundamental flavors of Dirac fermions.

\subsection{Comparison of the coupling $g_{\mathrm{VPP}}$ with the KSRF relation}

The $V \rightarrow P P$ coupling is often determined phenomenologically via the KSRF relation $g_{\mathrm{VPP}}^{\mathrm{KSRF}}=m_{\mathrm{V}} / F_{\mathrm{PS}}$ based on the vector-meson dominance $[32,33]$. This relation gives us

$$
g_{\mathrm{VPP}}^{\mathrm{KSRF}}=m_{V}^{\text {naive }} /\left(Z_{A} F_{P S}^{\text {bare }}\right)=9.4(6)
$$

at finite $m_{\mathrm{PS}}, V=24^{3} \times 48$ and $Z_{A}$ provided in section 2 . The central value $g_{\mathrm{VPP}}^{\mathrm{KSRF}}$ is $20 \%$ larger than the value $g_{\mathrm{VPP}}(3.12)$ rigorously extracted from the scattering.

Note furthermore that using our previous calculation with an unimproved action [26], and using the value of $m_{\mathrm{V}}$ and $F_{\mathrm{PS}}$ extrapolated to the chiral and continuum limit, we found $g_{\mathrm{VPP}}^{\mathrm{KSRF}}=13.1(2.2)$. The value $g_{\mathrm{VPP}}^{\mathrm{KSRF}}=15.6(4)$ used in [34] is from an update analysis including more gauge ensembles [55]. Our calculation therefore suggests that the KSRF relation overestimates the value of the coupling $g_{\mathrm{VPP}}$ for $\mathrm{SU}(2)$ gauge theory with two fundamental flavors of Dirac fermions and that the control of systematics must be improved in order to clarify the overal picture as a function of the number of flavours.

\section{Conclusions}

We determined the coupling $g_{\mathrm{VPP}}$ between a vector resonance and a pair of Goldstone bosons in a minimal realization of a composite Higgs model. This is the first lattice result for the coupling of a decaying resonance in a such 4-dimensional gauge theories with fermions. The calculation is performed by considering the scattering of two Goldstone 
bosons in the channel of the vector resonance. Assuming that the chiral dependence of $g_{\mathrm{VPP}}$ is mild (like in QCD) and that the discretization error are negligible, we obtain a rigorous estimate of the coupling in the continuum and in the chiral limit based on the non-perturbative calculation. The result suggests a rather large value of the coupling but smaller than the one obtained using the KSRF relation. Additional numerical simulations would be required to obtain a more definite answer. The coupling is relevant to constrain the phenomenology of a vector resonance in composite Higgs model at the LHC, and bring additional constraints on Vector Boson Scattering and on electroweak precision tests.

\section{Acknowledgments}

We would like to thank D. Buarque Franzosi, G. Cacciapaglia and F. Sannino for useful discussions at various stages of this project. The initial steps of this project have been performed on the HPC facilities at the HPCC centre of the University of Plymouth. This work was performed using the Cambridge Service for Data Driven Discovery (CSD3), part of which is operated by the University of Cambridge Research Computing on behalf of the STFC DiRAC HPC Facility (www.dirac.ac.uk). The DiRAC component of CSD3 was funded by BEIS capital funding via STFC capital grants ST/P002307/1 and ST/R002452/1 and STFC operations grant ST/R00689X/1. DiRAC is part of the National e-Infrastructure. S. P. was supported by Slovenian Research Agency ARRS (research core funding No. P1-0035 and No. J1-8137) and DFG grant No. SFB/TRR 55.

\section{A Conventions}

We follow the convention used in [51], where

$$
\sigma_{1}=\left(\begin{array}{ll}
0 & 1 \\
1 & 0
\end{array}\right), \quad \sigma_{2}=\left(\begin{array}{rr}
0 & -i \\
i & 0
\end{array}\right), \quad \sigma_{3}=\left(\begin{array}{rr}
1 & 0 \\
0 & -1
\end{array}\right), \quad \sigma_{4}=\left(\begin{array}{cc}
1 & 0 \\
0 & 1
\end{array}\right), \quad E=\left(\begin{array}{rc}
0 & \sigma_{4} \\
-\sigma_{4} & 0
\end{array}\right)
$$

and

$$
B_{1}=\sigma_{4}, B_{2}=i \sigma_{4}, B_{3}=\sigma_{3}, B_{4}=i \sigma_{3}, B_{5}=\sigma_{1}, B_{6}=i \sigma_{1}, \quad D_{4}=\sigma_{2}, D_{5}=i \sigma_{2} .
$$

The fifteen generators of SU(4) are denoted $S^{a}$ and $X^{i}$ with $a=1, \ldots, 10$ and $i=1, \ldots 5$. The generators $S^{a}$ satisfy the relation $\left(S^{a}\right)^{T} E+E S^{a}=0$ and are a representation of $\operatorname{Sp}(4)$ :

$$
\begin{aligned}
S^{a}=\frac{1}{2 \sqrt{2}}\left(\begin{array}{cc}
\sigma_{i} & 0 \\
0 & -\sigma_{i}^{T}
\end{array}\right), & a=1, \ldots, 4 & S^{a}=\frac{1}{2 \sqrt{2}}\left(\begin{array}{cc}
0 & B_{i} \\
B_{i}^{\dagger} & 0
\end{array}\right), & a=5, \ldots, 10 \\
X^{i}=\frac{1}{2 \sqrt{2}}\left(\begin{array}{cc}
\sigma_{i} & 0 \\
0 & \sigma_{i}^{T}
\end{array}\right), & i=1, \ldots, 3 & X^{i}=\frac{1}{2 \sqrt{2}}\left(\begin{array}{cc}
0 & D_{i} \\
D_{i}^{\dagger} & 0
\end{array}\right), & i=4,5 .
\end{aligned}
$$

The generators are normalized so that:

$$
\operatorname{tr}\left[S^{a} S^{b}\right]=\frac{1}{2} \delta^{a b}, \quad \operatorname{tr}\left[X^{i} X^{j}\right]=\frac{1}{2} \delta^{i j}, \quad \operatorname{tr}\left[S^{a} X^{i}\right]=0 .
$$

The structure constant of the algebra of $\operatorname{Sp}(4)$ are defined as $f_{a b c}=2 \operatorname{tr}\left[S^{a}\left[S^{b}, S^{c}\right]\right]$. 
The 10 generators of $\mathrm{SO}(5)$ with normalization $\operatorname{Tr}\left[T^{i} T^{j}\right]=\frac{1}{2} \delta^{i j}$ are defined as follows:

$$
\begin{aligned}
& T^{1}=\frac{i}{2}\left(\begin{array}{ccccc}
0 & 0 & 0 & 0 & 0 \\
0 & 0 & 1 & 0 & 0 \\
0 & -1 & 0 & 0 & 0 \\
0 & 0 & 0 & 0 & 0 \\
0 & 0 & 0 & 0 & 0
\end{array}\right) \quad T^{2}=\frac{i}{2}\left(\begin{array}{ccccc}
0 & 0 & 1 & 0 & 0 \\
0 & 0 & 0 & 0 & 0 \\
-1 & 0 & 0 & 0 & 0 \\
0 & 0 & 0 & 0 & 0 \\
0 & 0 & 0 & 0 & 0
\end{array}\right) \quad T^{3}=\frac{i}{2}\left(\begin{array}{ccccc}
0 & 1 & 0 & 0 & 0 \\
-1 & 0 & 0 & 0 & 0 \\
0 & 0 & 0 & 0 & 0 \\
0 & 0 & 0 & 0 & 0 \\
0 & 0 & 0 & 0 & 0
\end{array}\right) \quad T^{4}=\frac{i}{2}\left(\begin{array}{ccccc}
0 & 0 & 0 & 0 & 0 \\
0 & 0 & 0 & 0 & 0 \\
0 & 0 & 0 & 0 & 0 \\
0 & 0 & 0 & 0 & -1 \\
0 & 0 & 0 & 1 & 0
\end{array}\right) \\
& T^{5}=\frac{i}{2}\left(\begin{array}{ccccc}
0 & 0 & 0 & 0 & 0 \\
0 & 0 & 0 & 0 & 1 \\
0 & 0 & 0 & 0 & 0 \\
0 & 0 & 0 & 0 & 0 \\
0 & -1 & 0 & 0 & 0
\end{array}\right) \quad T^{6}=\frac{i}{2}\left(\begin{array}{ccccc}
0 & 0 & 0 & 0 & 0 \\
0 & 0 & 0 & 1 & 0 \\
0 & 0 & 0 & 0 & 0 \\
0 & -1 & 0 & 0 & 0 \\
0 & 0 & 0 & 0 & 0
\end{array}\right) \quad T^{7}=\frac{i}{2}\left(\begin{array}{ccccc}
0 & 0 & 0 & 1 & 0 \\
0 & 0 & 0 & 0 & 0 \\
0 & 0 & 0 & 0 & 0 \\
-1 & 0 & 0 & 0 & 0 \\
0 & 0 & 0 & 0 & 0
\end{array}\right) \quad T^{8}=\frac{i}{2}\left(\begin{array}{ccccc}
0 & 0 & 0 & 0 & -1 \\
0 & 0 & 0 & 0 & 0 \\
0 & 0 & 0 & 0 & 0 \\
0 & 0 & 0 & 0 & 0 \\
1 & 0 & 0 & 0 & 0
\end{array}\right) \\
& T^{9}=\frac{i}{2}\left(\begin{array}{ccccc}
0 & 0 & 0 & 0 & 0 \\
0 & 0 & 0 & 0 & 0 \\
0 & 0 & 0 & -1 & 0 \\
0 & 0 & 1 & 0 & 0 \\
0 & 0 & 0 & 0 & 0
\end{array}\right) \quad T^{10}=\frac{i}{2}\left(\begin{array}{ccccc}
0 & 0 & 0 & 0 & 0 \\
0 & 0 & 0 & 0 & 0 \\
0 & 0 & 0 & 0 & 1 \\
0 & 0 & 0 & 0 & 0 \\
0 & 0 & -1 & 0 & 0
\end{array}\right) .
\end{aligned}
$$

Open Access. This article is distributed under the terms of the Creative Commons Attribution License (CC-BY 4.0), which permits any use, distribution and reproduction in any medium, provided the original author(s) and source are credited.

\section{References}

[1] D.B. Kaplan and H. Georgi, $\mathrm{SU}(2) \times \mathrm{U}(1)$ breaking by vacuum misalignment, Phys. Lett. $B$ 136 (1984) 183 [INSPIRE].

[2] D.B. Kaplan, H. Georgi and S. Dimopoulos, Composite Higgs scalars, Phys. Lett. B 136 (1984) 187 [INSPIRE].

[3] M.J. Dugan, H. Georgi and D.B. Kaplan, Anatomy of a composite Higgs model, Nucl. Phys. $B \mathbf{2 5 4}$ (1985) 299 [INSPIRE].

[4] R. Contino and M. Salvarezza, One-loop effects from spin-1 resonances in composite Higgs models, JHEP 07 (2015) 065 [arXiv: 1504.02750] [INSPIRE].

[5] R. Contino, D. Marzocca, D. Pappadopulo and R. Rattazzi, On the effect of resonances in composite Higgs phenomenology, JHEP 10 (2011) 081 [arXiv:1109.1570] [INSPIRE].

[6] M. Gallinaro et al., Beyond the Standard Model in vector boson scattering signatures, in International workshop on BSM models in vector boson scattering processes, (2020) [arXiv: 2005. 09889] [INSPIRE].

[7] D. Liu, L.-T. Wang and K.-P. Xie, Broad composite resonances and their signals at the LHC, Phys. Rev. D 100 (2019) 075021 [arXiv:1901.01674] [InSPIRE].

[8] C. Helsens, D. Jamin, M.L. Mangano, T.G. Rizzo and M. Selvaggi, Heavy resonances at energy-frontier hadron colliders, Eur. Phys. J. C 79 (2019) 569 [arXiv:1902.11217] [INSPIRE]. 
[9] D. Buarque Franzosi, G. Cacciapaglia and A. Deandrea, Sigma-assisted low scale composite Goldstone-Higgs, Eur. Phys. J. C 80 (2020) 28 [arXiv:1809.09146] [INSPIRE].

[10] D. Liu, L.-T. Wang and K.-P. Xie, Prospects of searching for composite resonances at the LHC and beyond, JHEP 01 (2019) 157 [arXiv:1810.08954] [INSPIRE].

[11] D. Buarque Franzosi, Implications of vector boson scattering unitarity in composite Higgs models, PoS (EPS-HEP2017) 264 (2017).

[12] D. Greco and D. Liu, Hunting composite vector resonances at the LHC: naturalness facing data, JHEP 12 (2014) 126 [arXiv: 1410. 2883] [INSPIRE].

[13] G. Cacciapaglia and F. Sannino, Fundamental composite (Goldstone) Higgs dynamics, JHEP 04 (2014) 111 [arXiv: 1402.0233] [INSPIRE].

[14] G. Cacciapaglia, C. Pica and F. Sannino, Fundamental composite dynamics: a review, Phys. Rept. 877 (2020) 1 [arXiv: 2002.04914] [InSPIRE].

[15] A. Arbey, G. Cacciapaglia, H. Cai, A. Deandrea, S. Le Corre and F. Sannino, Fundamental composite electroweak dynamics: status at the LHC, Phys. Rev. D 95 (2017) 015028 [arXiv: 1502.04718] [INSPIRE].

[16] D. Buarque Franzosi, G. Cacciapaglia, H. Cai, A. Deandrea and M. Frandsen, Vector and axial-vector resonances in composite models of the Higgs boson, JHEP 11 (2016) 076 [arXiv: 1605.01363] [INSPIRE].

[17] M. Lüscher, Two particle states on a torus and their relation to the scattering matrix, Nucl. Phys. B 354 (1991) 531 [INSPIRE].

[18] K. Rummukainen and S.A. Gottlieb, Resonance scattering phase shifts on a nonrest frame lattice, Nucl. Phys. B 450 (1995) 397 [hep-lat/9503028] [INSPIRE].

[19] CP-PACS collaboration, Lattice QCD calculation of the $\rho$ meson decay width, Phys. Rev. D 76 (2007) 094506 [arXiv:0708.3705] [INSPIRE].

[20] CS collaboration, $\rho$ meson decay in $2+1$ flavor lattice QCD, Phys. Rev. D 84 (2011) 094505 [arXiv:1106.5365] [INSPIRE].

[21] X. Feng, K. Jansen and D.B. Renner, Resonance parameters of the $\rho$-meson from lattice QCD, Phys. Rev. D 83 (2011) 094505 [arXiv: 1011.5288] [InSPIRE].

[22] C.B. Lang, D. Mohler, S. Prelovsek and M. Vidmar, Coupled channel analysis of the $\rho$ meson decay in lattice QCD, Phys. Rev. D 84 (2011) 054503 [Erratum ibid. 89 (2014) 059903] [arXiv: 1105.5636] [INSPIRE].

[23] Hadron Spectrum collaboration, Energy dependence of the $\rho$ resonance in $\pi \pi$ elastic scattering from lattice QCD, Phys. Rev. D 87 (2013) 034505 [Erratum ibid. 90 (2014) 099902] [arXiv:1212.0830] [INSPIRE].

[24] F. Erben, J.R. Green, D. Mohler and H. Wittig, Rho resonance, timelike pion form factor, and implications for lattice studies of the hadronic vacuum polarization, Phys. Rev. D 101 (2020) 054504 [arXiv:1910.01083] [INSPIRE].

[25] C. Alexandrou et al., P-wave $\pi \pi$ scattering and the $\rho$ resonance from lattice $Q C D$, Phys. Rev. D 96 (2017) 034525 [arXiv: 1704.05439] [INSPIRE].

[26] R. Arthur, V. Drach, M. Hansen, A. Hietanen, C. Pica and F. Sannino, SU(2) gauge theory with two fundamental flavors: a minimal template for model building, Phys. Rev. D 94 (2016) 094507 [arXiv: 1602.06559] [INSPIRE]. 
[27] R. Arthur, V. Drach, A. Hietanen, C. Pica and F. Sannino, SU(2) gauge theory with two fundamental flavours: scalar and pseudoscalar spectrum, arXiv:1607.06654 [INSPIRE].

[28] R. Arthur, V. Drach, M. Hansen, A. Hietanen, C. Pica and F. Sannino, Scattering lengths in $\mathrm{SU}(2)$ gauge theory with two fundamental fermions, PoS (LATTICE2014) 271 (2014) [arXiv: 1412.4771] [INSPIRE].

[29] V. Drach, A. Hietanen, C. Pica, J. Rantaharju and F. Sannino, Template composite dark matter: SU(2) gauge theory with 2 fundamental flavours, PoS(LATTICE2015) 234 (2016) [arXiv: 1511.04370] [INSPIRE].

[30] A. Hietanen, R. Lewis, C. Pica and F. Sannino, Fundamental composite Higgs dynamics on the lattice: SU(2) with two flavors, JHEP 07 (2014) 116 [arXiv: 1404.2794] [INSPIRE].

[31] A. Hietanen, R. Lewis, C. Pica and F. Sannino, Composite Goldstone dark matter: experimental predictions from the lattice, JHEP 12 (2014) 130 [arXiv:1308.4130] [INSPIRE].

[32] K. Kawarabayashi and M. Suzuki, Partially conserved axial vector current and the decays of vector mesons, Phys. Rev. Lett. 16 (1966) 255 [INSPIRE].

[33] Riazuddin and Fayyazuddin, Algebra of current components and decay widths of $\rho$ and $K^{*}$ mesons, Phys. Rev. 147 (1966) 1071 [InSPIRE].

[34] D. Nogradi and L. Szikszai, The model dependence of $m_{\varrho} / f_{\pi}$, PoS (LATTICE2019) 237 (2019) [arXiv: 1912.04114] [INSPIRE].

[35] D. Nogradi and L. Szikszai, The flavor dependence of $m_{\varrho} / f_{\pi}$, JHEP 05 (2019) 197 [arXiv: 1905.01909] [INSPIRE].

[36] E. Bennett et al., $\mathrm{Sp}(4)$ gauge theories on the lattice: quenched fundamental and antisymmetric fermions, Phys. Rev. D 101 (2020) 074516 [arXiv: 1912.06505] [INSPIRE].

[37] E. Bennett et al., $\mathrm{Sp}(4)$ gauge theories on the lattice: $N_{f}=2$ dynamical fundamental fermions, JHEP 12 (2019) 053 [arXiv: 1909.12662] [INSPIRE].

[38] V. Ayyar et al., Spectroscopy of $\mathrm{SU}(4)$ composite Higgs theory with two distinct fermion representations, Phys. Rev. D 97 (2018) 074505 [arXiv:1710.00806] [INSPIRE].

[39] Lattice Strong Dynamics collaboration, Nonperturbative investigations of SU(3) gauge theory with eight dynamical flavors, Phys. Rev. D 99 (2019) 014509 [arXiv:1807.08411] [INSPIRE].

[40] K.G. Wilson, Confinement of quarks, Phys. Rev. D 10 (1974) 2445 [inSPIRE].

[41] B. Sheikholeslami and R. Wohlert, Improved continuum limit lattice action for QCD with Wilson fermions, Nucl. Phys. B 259 (1985) 572 [INSPIRE].

[42] M. Lüscher and P. Weisz, On-shell improved lattice gauge theories, Commun. Math. Phys. 97 (1985) 59 [Erratum ibid. 98 (1985) 433] [INSPIRE].

[43] S. Borsányi et al., High-precision scale setting in lattice QCD, JHEP 09 (2012) 010 [arXiv: 1203.4469] [INSPIRE].

[44] O. Bär and M. Golterman, Chiral perturbation theory for gradient flow observables, Phys. Rev. D 89 (2014) 034505 [Erratum ibid. 89 (2014) 099905] [arXiv:1312.4999] [INSPIRE].

[45] G. Martinelli, C. Pittori, C.T. Sachrajda, M. Testa and A. Vladikas, A general method for nonperturbative renormalization of lattice operators, Nucl. Phys. B 445 (1995) 81 [hep-lat/9411010] [INSPIRE]. 
[46] M. Gockeler et al., Nonperturbative renormalization of composite operators in lattice QCD, Nucl. Phys. B 544 (1999) 699 [hep-lat/9807044] [INSPIRE].

[47] P.F. Bedaque, Aharonov-Bohm effect and nucleon nucleon phase shifts on the lattice, Phys. Lett. B 593 (2004) 82 [nucl-th/0402051] [INSPIRE].

[48] C.T. Sachrajda and G. Villadoro, Twisted boundary conditions in lattice simulations, Phys. Lett. B 609 (2005) 73 [hep-lat/0411033] [INSPIRE].

[49] L. Del Debbio, M.T. Frandsen, H. Panagopoulos and F. Sannino, Higher representations on the lattice: perturbative studies, JHEP 06 (2008) 007 [arXiv:0802.0891] [INSPIRE].

[50] M. Lüscher, Signatures of unstable particles in finite volume, Nucl. Phys. B 364 (1991) 237 [INSPIRE].

[51] T.A. Ryttov and F. Sannino, Ultra minimal technicolor and its dark matter TIMP, Phys. Rev. D 78 (2008) 115010 [arXiv:0809.0713] [INSPIRE].

[52] C. Michael, Adjoint sources in lattice gauge theory, Nucl. Phys. B 259 (1985) 58 [InSPIRE].

[53] M. Lüscher and U. Wolff, How to calculate the elastic scattering matrix in two-dimensional quantum field theories by numerical simulation, Nucl. Phys. B 339 (1990) 222 [INSPIRE].

[54] B. Blossier, M. Della Morte, G. von Hippel, T. Mendes and R. Sommer, On the generalized eigenvalue method for energies and matrix elements in lattice field theory, JHEP 04 (2009) 094 [arXiv: 0902.1265] [INSPIRE].

[55] V. Drach, T. Janowski and C. Pica, Update on SU(2) gauge theory with $N_{F}=2$ fundamental flavours, EPJ Web Conf. 175 (2018) 08020 [arXiv:1710.07218] [INSPIRE]. 